\title{
Measurement of endometrial thickness by transvaginal ultrasonography to predict pathological response to medroxyprogesterone acetate in patients with grade 1 endometrioid adenocarcinoma
}

\author{
MASAKAZU SATO, TAKAHIDE ARIMOTO, KEI KAWANA, YUICHIRO MIYAMOTO, YUJI IKEDA, \\ KENSUKE TOMIO, MICHIHIRO TANIKAWA, KENBUN SONE, MAYUYO MORI-UCHINO, TETSUSHI TSURUGA, \\ KAZUNORI NAGASAKA, KATSUYUKI ADACHI, YOKO MATSUMOTO, \\ KATSUTOSHI ODA, YUTAKA OSUGA and TOMOYUKI FUJII
}

Department of Obstetrics and Gynecology, Faculty of Medicine, University of Tokyo, Tokyo 1138655, Japan

Received July 9, 2015; Accepted November 16, 2015

DOI: $10.3892 / \mathrm{mco} .2016 .748$

\begin{abstract}
The aim of the present study was to evaluate whether measuring endometrial thickness during fertility-sparing treatment with medroxyprogesterone acetate (MPA) can be a predictive marker for effectiveness in women with endometrioid adenocarcinoma, grade 1 (EmCa, G1). A total of 32 patients with stage IA EmCa, G1 underwent treatment with MPA. Patients were $<40$ years of age and preferred fertility-sparing treatment. MPA (600 mg/day) with low-dose aspirin was administered orally for 26 weeks. Pathological evaluation was performed by total curettage at weeks 8 and 16 and by fractional curettage at week 26. Patients underwent curative surgery in case of disease progression. Endometrial thickness was measured by transvaginal ultrasonography at weeks 8 and 16. Patients who showed non-complete response (non-CR) had thicker endometrium than that of $\mathrm{CR}$ patients at weeks 8 and 16. Receiver operating characteristic analysis revealed cut-off values of 8.3 and $4.7 \mathrm{~mm}$ endometrial thickness at weeks 8 and 16, respectively, for non-CR. Endometrial thickness $>5 \mathrm{~mm}$ at week 16 was an independent factor for prediction of non-CR. Measurement of endometrial thickness during MPA treatment may be useful as a predictive marker for pathological response to MPA in patients with EmCa, G1.
\end{abstract}

Correspondence to: Dr Kei Kawana, Department of Obstetrics and Gynecology, Faculty of Medicine, University of Tokyo, 7-3-1 Hongo, Tokyo 1138655, Japan

E-mail:kkawana-tky@umin.org

Key words: endometrioid carcinoma, medroxyprogesterone acetate, fertility-sparing treatment, transvaginal ultrasonography, endometrial thickness

\section{Introduction}

Endometrial cancer is one of the most common gynecological malignancies. The standard treatment for early-stage endometrial cancer is primarily total abdominal hysterectomy and bilateral salpingo-oophorectomy, with additional pelvic and para-aortic lymph node dissection (1). By contrast, preservation of fertility for early-stage endometrial cancer has become more important due to the increasing age of first parity in recent years. Use of fertility-sparing approaches, such as hormonal therapies using medroxyprogesterone acetate (MPA) or megestrol acetate, gonadotropin-releasing hormone-agonist, and intrauterine devices, which release levonorgestrel, have been reported (2-9).

Of these, treatment with MPA is commonly used as a fertility-sparing approach in Japan, and various studies have shown its efficacy (10-13). It has been reported that the complete response (CR) rate for atypical endometrial hyperplasia by initial treatment with MPA was $>80 \%$. However, only $55-75 \%$ of patients with grade 1 endometrioid adenocarcinoma (EmCa, G1) treated by MPA achieved CR. Currently, frequent pathological inspections are required to evaluate the efficacy of MPA treatment; however, repeated endometrial curettages are invasive, and can reduce fertility by injury to the endometrium $(14,15)$. Therefore, establishment of biological markers predicting the efficacy of MPA is required.

However, there have been few studies that indicate the predictive factors of the success of hormonal therapies other than histological markers, such as estrogen and progesterone receptor (ER, PgR) expression $(16,17)$. Although sensitivity to MPA is thought to be associated with the presence of ER and $\mathrm{PgR}$, it is known that patients with receptor-positive tumors do not necessarily respond completely to MPA.

Measurement of endometrial thickness using transvaginal ultrasonography (TVUS) is a convenient and widely-used technique, and can be used as a non-invasive screening test for detection of endometrial cancer. Endometrium thicker than 
5 or $20 \mathrm{~mm}$ for post- or pre-menopausal women, respectively, has been shown to be a clinically useful predictive marker for endometrial cancer (18).

Considering the universal use of TVUS and the clinical significance of endometrial thickness, measurement of changes in endometrial thickness during treatment may be used as a non-invasive and useful predictive marker for the therapeutic efficacy of MPA therapy. In the present study, endometrial thickness was measured by TVUS during MPA therapy and its association with pathological response to MPA was assessed.

\section{Materials and methods}

Patients. The study was approved by the institutional ethics committee (The University of Tokyo, Tokyo, Japan). Medical records and data from the gynecological oncology database of patients who had undergone treatment with MPA at the University of Tokyo Hospital between 1999 and 2012 were retrospectively reviewed.

Patients were deemed suitable for this fertility-sparing treatment provided they met the following criteria: i) Age $<40$ years, ii) pathological diagnosis of endometrioid endometrial adenocarcinoma with grade 1 differentiation (EmCa, G1), iii) absence of myometrial invasion and extrauterine spread on magnetic resonance imaging, and iv) a desire of the patient to preserve their fertility and undergo hormonal therapy following a detailed discussion regarding the current standard treatment and requirement for follow-up with pathological examinations.

MPA therapy and clinical assessment. All the patients who met the inclusion criteria were administered MPA (600 mg/day with 81-100 mg of aspirin) orally, and this was continued for 26 weeks. As an interim evaluation, pathological response to MPA was assessed by histological examination at weeks 8 and 16 of the treatment period by performing dilatation and total curettage (D\&C). The histological diagnoses were confirmed by an experienced pathologist (or central pathological review if it was difficult to diagnose). As an end-point, total or fractional curettage was performed at week 26 of the treatment for histological assessment. A CR was defined as the status with evidence of neither carcinoma nor hyperplasia at week 26 of the treatment.

Measurement of endometrial thickness by TVUS. Endometrial thickness was measured using TVUS before D\&C at weeks 8 and 16 of the treatment. The measuring procedure was as follows: i) Detect the uterus in sagittal view, ii) detect the mid-line view where the endometrium was visible from the fundus to the cervix of the uterus in succession, iii) keep the line perpendicular to the mid-line, and iv) measure the endometrium by keeping the endpoints of the line positioned on the outer edge of each side of the endometrium (13).

Statistical analysis. Logistic regression was used to calculate any statistical differences. Receiver operating characteristic (ROC) curves were used to assess the criterion value. $\mathrm{P}<0.05$ was considered to indicate a statistically significant difference. JMP ${ }^{\circledR}$ (SAS Institute, Inc., Cary, NC, USA) was used for statistical analysis.
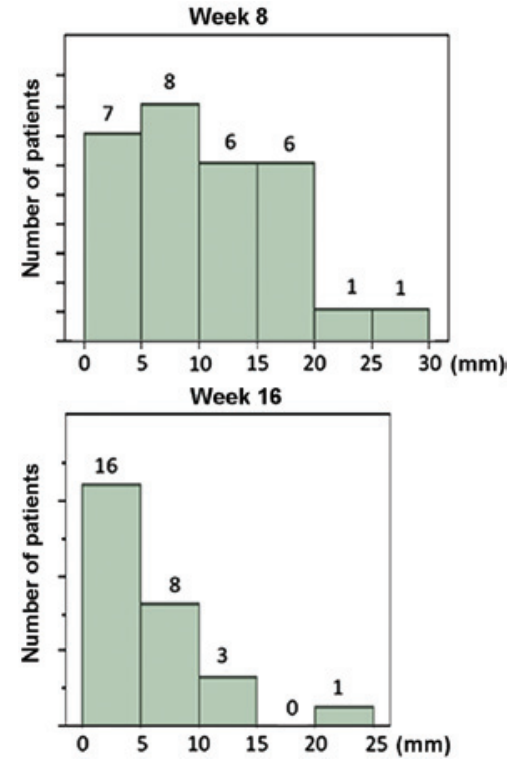

Figure 1. Endometrial thickness at weeks 8 and 16 during medroxyprogesterone acetate (MPA) therapy. The endometrium tended to become thinner with continuing MPA therapy. Endometrium thickness is shown on the $\mathrm{x}$-axis.

\section{Results}

Patient characteristics. A total of 32 patients were enrolled in the present study. Patient backgrounds are described as follows. The mean age at initiation of treatment was 33 years (range, 19-39 years). Of them, 31 (97\%) patients were nulliparous. The mean body mass index (BMI) was $20.1 \mathrm{~kg} / \mathrm{m}^{2}$ (range, 17.1-37.4 kg/m²) . Six (19\%) patients had polycystic ovary syndrome. One (3\%) patient was positive for carbohydrate antigen 125 (CA125) and 3 (9\%) for CA19-9.

CR for patients. CR was achieved in 19 (59\%) patients with carcinoma. The remaining 13 were non-CR patients, of whom seven underwent curative surgery (hysterectomy + bilateral salpingo-oophorectomy) at week 16, and four patients at week 26 of treatment. Two patients refused to undergo curative surgery despite not achieving CR by week 26 of treatment, and was followed by the administration of oral estrogen-progestin treatment for at least 6 months, resulting in no evidence of malignancy.

Endometrial thickness. The distribution of endometrial thickness at weeks 8 and 16 of treatment are shown in Fig. 1; all data were included in this analysis. With continuing MPA therapy, the endometrium tended to become thinner. The regression line for endometrial thickness at weeks 8 and 16 is shown in Fig. 2, with a correlation coefficient of 0.62. Non-CR patients had significantly thicker endometrium than that of $\mathrm{CR}$ patients at weeks 8 and 16 of treatment. Changes in endometrial thickness at weeks 8 and 16 in the same patient are shown in Fig. 3. CR patients tended to have a more notable reduction of endometrial thickness from week 8 to week 16 , which may be attributable to the MPA therapy.

Cut-off. The cut-off points were chosen based on the ROC curve: $8.3 \mathrm{~mm}$ with a sensitivity/specificity of $0.92 / 0.63$ at week 8 , and 
Table I. Predictive factors of non-CR with MPA therapy.

\begin{tabular}{|c|c|c|c|c|}
\hline \multirow[b]{2}{*}{ Characteristics } & \multicolumn{2}{|c|}{ Univariate } & \multicolumn{2}{|c|}{ Multivariate } \\
\hline & Odds ratio $(95 \% \mathrm{CI})$ & P-value & Odds ratio $(95 \% \mathrm{CI})$ & P-value \\
\hline Age $>30$ years & $4.95(0.85-28.6)$ & 0.0533 & $6.68(0.57-184.54)$ & 0.1336 \\
\hline$>8 \mathrm{~mm}$ endometrium at week 8 & $15.4(1.59-148)$ & $0.0038^{\mathrm{a}}$ & - & - \\
\hline$>5 \mathrm{~mm}$ endometrium at week 16 & $19.5(2.69-141)$ & $0.0008^{\mathrm{a}}$ & $20.52(3.05-231.16)$ & $0.0012^{\mathrm{a}}$ \\
\hline $\mathrm{BMI}>25$ & $2.62(0.35-19.1)$ & 0.3356 & - & - \\
\hline PCOS positive & $0.68(0.105-4.41)$ & 0.6839 & - & - \\
\hline
\end{tabular}

${ }^{a} \mathrm{P}<0.05$. Multivariate analysis showed that endometrial thickness at week 16 of treatment was an independent predictive marker for final non-CR by week 16. CR, complete response; MPA, medroxyprogesterone acetate; BMI, body mass index; PCOS, polycystic ovarian syndrome.

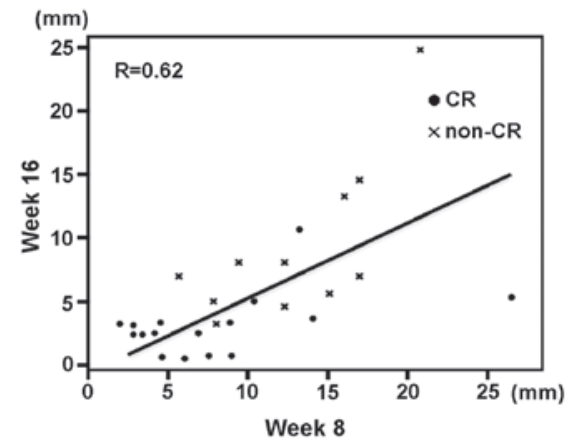

Figure 2. Correlation between thickness of endometrium at weeks 8 and 16. Patients who had a thicker endometrium at week 8 also had thicker endometrium at week 16. Non-CR patients had thicker endometrium than that of CR patients at weeks 8 and 16 . Weeks 8 and 16 endometrium thickness are shown on the $\mathrm{x}$ - and $\mathrm{y}$-axis, respectively. $\mathrm{CR}$, complete response.
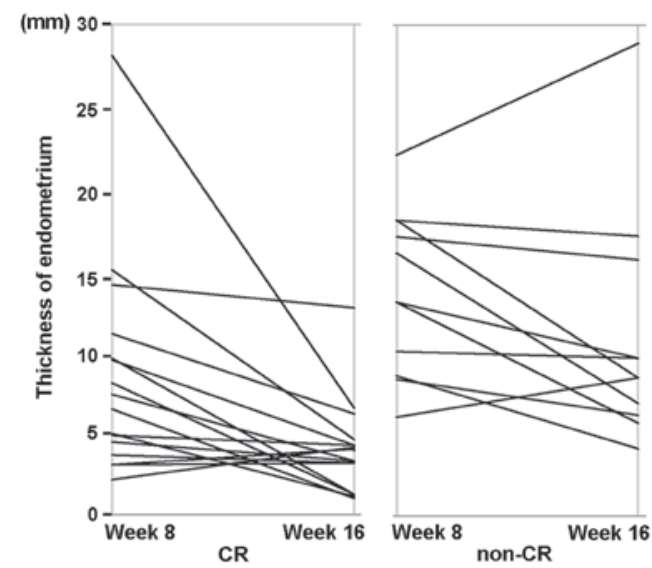

Figure 3. Change in endometrial thickness from week 8 to 16 in the same patient. CR patients tended to have a more notable reduction of endometrial thickness from week 8 to 16 , which may be attributable to the medroxyprogesterone acetate therapy. $\mathrm{CR}$, complete response.

$4.7 \mathrm{~mm}$ with a sensitivity/specificity of $0.91 / 0.81$ at week 16 (Fig. 4). Therefore, the values of 8 and $5 \mathrm{~mm}$ for weeks 8 and 16, respectively, were chosen as representative values for clinical significance and easiness to apply in daily practice.

Univariate analysis. Univariate analysis revealed that endometrial thickness at weeks 8 and 16 of MPA therapy
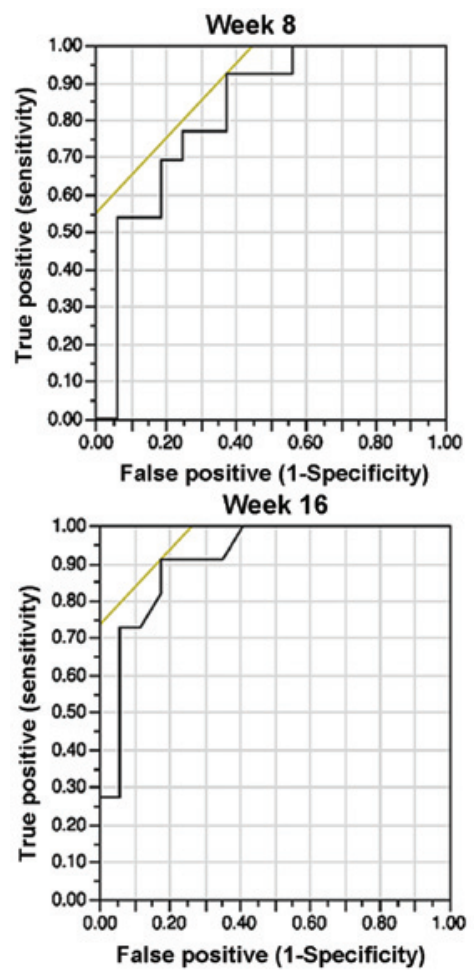

Figure 4. Receiver operating characteristic curves of endometrium thickness at weeks 8 and 16. Cut-off points for non-complete response were chosen: $8.3 \mathrm{~mm}$ with a sensitivity/specificity of $0.92 / 0.63$ at week 8 and $4.7 \mathrm{~mm}$ with a sensitivity/specificity of $0.91 / 0.81$ at week 16 .

were significant predictive markers for non-CR by week 26 . Furthermore, multivariate analysis demonstrated that endometrial thickness at week 16 of treatment was an independent predictive marker for final non-CR by week 16 (Table I).

\section{Discussion}

Fertility-sparing approaches are often sought for younger patients with early-stage endometrial cancer. In the present study, endometrial thickness at weeks 8 and 16 of MPA treatment in patients with EmCa, G1 showed significant correlation with pathological response to MPA; thicker endometrium (>8 $\mathrm{mm}$ at week 8 or $>5 \mathrm{~mm}$ at week 16) suggested a higher likelihood of non-CR by the end-point (week 26). These results 

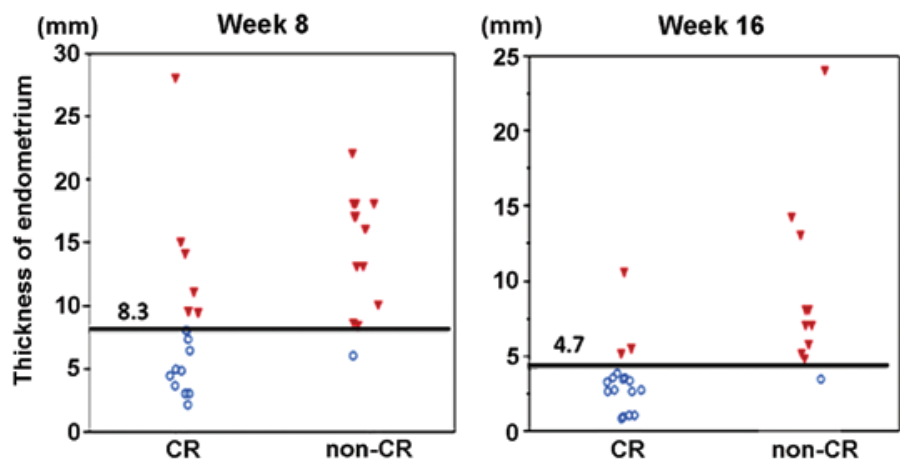

Figure 5. Endometrial thickness at weeks 8 and 16 in patients who showed CR and non-CR, with cut-offs determined by the receiver operating characteristic curves. Endometrial thickness at week 16 was a more useful predictive marker for pathological non-CR than that at week 8. CR, complete response.

are plausible in the context of daily clinical practice and is in accordance with a previous study (13). To the best of our knowledge, only the study by Ushijima et al (13) has suggested the usefulness of TVUS for assessment of response to MPA treatment. The study concluded that a thinner endometrium at 8 weeks compared to pretreatment thickness predicted a $73 \%$ possibility of achieving CR at 26 weeks, whereas a thicker endometrium at 8 weeks was predicted to have a $25 \%$ possibility of achieving CR at 26 weeks. In the present study, endometrial thickness at pretreatment was not assessed as certain patients had already undergone $\mathrm{D} \& \mathrm{C}$ prior to initial consultation, and their endometrium thickness was thought to be thinner than prior to curettage.

The present results suggested that endometrial thickness at weeks 8 and 16 in the same patient tended to be correlated. In all cases, no clear residual endometrium was observed by TVUS after each D\&C, and thereby endometrial thickness at weeks 8 and 16 was thought to not be influenced by residual tissues prior to D\&C. In addition, the absolute change of thickness from weeks 8 to 16 was not correlated with pathological response ( $\mathrm{CR}$ or non- $\mathrm{CR})(\mathrm{P}=0.861$, data not shown). The reciprocal change of endometrial thickness at weeks 8 and 16 may suggest that endometrial thickness reflects the continuous suppression of cancer expansion by MPA.

Endometrial thickness at week 16 was a more useful predictive marker for pathological non-CR than that at week 8 , although the thicknesses at weeks 8 and 16 were correlated (Fig. 5). Endometrial thickness at week 8 may be influenced by thickness prior to MPA therapy, while thickness at week 16 was not likely to be so due to complete dissection of the endometrium by $\mathrm{D \& C}$ at week 8 . In certain patients, thickness had decreased significantly between weeks 8 and 16 .

The most reliable marker for clinical efficacy of MPA in EmCa patients is a histological approach by total curettage $(\mathrm{D} \& \mathrm{C})$. In the present series, certain patients showed an unexpected outcome association with endometrial thickness. One patient had an endometrial thickness of $28 \mathrm{~mm}(>8 \mathrm{~mm})$ at week 8 and $5.4 \mathrm{~mm}(>5 \mathrm{~mm})$ at week 16; however, the patient exhibited pathological CR by the end-point. Another patient had an endometrial thickness of $3.4 \mathrm{~mm}(<5 \mathrm{~mm})$ at week 16 , but did not show pathological CR at the end-point (Fig. 3). Therefore, the present data cannot conclude that TVUS can be used as a complete substitute for biopsy or D\&C. These results warrant a future prospective study, in which patients with endometrium thicker than $8 \mathrm{~mm}$ at week 8 or $5 \mathrm{~mm}$ at week 16 of MPA treatment are recommended to undergo D\&C at each time point, while the remaining patients undergo D\&C at the end-point of the treatment.

In conclusion, endometrial thickness by TVUS during MPA therapy (at week 16) can be a predictive marker for pathological response to MPA in patients with EmCa, G1. Considering the side effects of multiple D\&C procedures on reproduction, non-invasive TVUS for endometrial thickness assessment may be useful as primary screening for efficacy of MPA therapy, and may assist in the decision making process of whether D\&C should be performed or not.

\section{References}

1. Pecorelli S: Revised FIGO staging for carcinoma of the vulva, cervix and endometrium. Int J Gynaecol Obstet 105: 103-104, 2009.

2. Park H, Seok JM, Yoon BS, Seong SJ, Kim JY, Shim JY and Park CT: Effectiveness of high-dose progestin and long-term outcomes in young women with early-stage, well-differentiated endometrioid adenocarcinoma of uterine endometrium. Arch Gynecol Obstet 285: 473-478, 2012.

3. Kalogiannidis I and Agorastos T: Conservative management of young patients with endometrial highly-differentiated adenocarcinoma. J Obstet Gynaecol 31: 13-17, 2011.

4. Laurelli G, Di Vagno G, Scaffa C, Losito S, Del Giudice M and Greggi S: Conservative treatment of early endometrial cancer: Preliminary results of a pilot study. Gynecol Oncol 120: 43-46, 2011.

5. Bovicelli A, D'Andrilli G, Giordano A and De Iaco P: Conservative treatment of early endometrial cancer. J Cell Physiol 228: 1154-1158, 2013.

6. Morelli M, Di Cello A, Venturella R, Mocciaro R, D'Alessandro P and Zullo F: Efficacy of the levonorgestrel intrauterine system (LNG-IUS) in the prevention of the atypical endometrial hyperplasia and endometrial cancer: Retrospective data from selected obese menopausal symptomatic women. Gynecol Endocrinol 29: 156-159, 2013.

7. Cade TJ, Quinn MA, Rome RM and Neesham D: Progestogen treatment options for early endometrial cancer. BJOG 117: 879-884, 2010.

8. Ramirez PT, Frumovitz M, Bodurka DC, Sun CC and Levenback C: Hormonal therapy for the management of grade 1 endometrial adenocarcinoma: A literature review. Gynecol Oncol 95: 133-138, 2004.

9. Gunderson CC, Fader AN, Carson KA and Bristow RE: Oncologic and reproductive outcomes with progestin therapy in women with endometrial hyperplasia and grade 1 adenocarcinoma: A systematic review. Gynecol Oncol 125: 477-482, 2012.

10. Chang WH, Chen $\mathrm{CH}$ and $\mathrm{Yu} \mathrm{MH}$ : Conservative therapy of stage I endometrial adenocarcinoma and atypical endometrial hyperplasia for the preservation of fertility. Int $\mathbf{J}$ Gynaecol Obstet 92: 137-138, 2006 
11. Fujiwara H, Jobo T, Takei Y, Saga Y, Imai M, Arai T, Taneichi A, Machida S, Takahashi Y and Suzuki M: Fertility-sparing treatment using medroxyprogesterone acetate for endometrial carcinoma. Oncol Lett 3: 1002-1006, 2012.

12. Yahata T, Fujita K, Aoki Y and Tanaka K: Long-term conservative therapy for endometrial adenocarcinoma in young women. Hum Reprod 21: 1070-1075, 2006.

13. Ushijima K, Yahata H, Yoshikawa H, Konishi I, Yasugi T, Saito T, Nakanishi T, Sasaki H, Saji F, Iwasaka T, et al: Multicenter phase II study of fertility-sparing treatment with medroxyprogesterone acetate for endometrial carcinoma and atypical hyperplasia in young women. J Clin Oncol 25: 2798-2803, 2007.

14. Fujimoto A, Ichinose M,Harada M, Hirata T, Osuga Y and Fujii T: The outcome of infertility treatment in patients undergoing assisted reproductive technology after conservative therapy for endometrial cancer. J Assit Repord Genet 31: 1189-1194, 2014.

15. Shufaro Y, Simon A, Laufer N and Fatum M: Thin unresponsive endometrium-a possible complication of surgical curettage compromising ART outcome. J Assit Repord Genet 25: 421-425, 2008 .
16. Yamazawa K, Hirai M, Fujito A, Nishi H, Terauchi F, Ishikura $H$, Shozu M and Isaka K: Fertility-preserving treatment with progestin and pathological criteria to predict responses, in young women with endometrial cancer. Hum Reprod 22: 1953-1958, 2007.

17. Minaguchi T, Nakagawa S, Takazawa Y, Nei T, Horie K, Fujiwara T, Osuga Y, Yasugi T, Kugu K, Yano T, et al: Combined phospho-Akt and PTEN expressions associated with post-treatment hysterectomy after conservative progestin therapy in complex atypical hyperplasia and stage Ia, G1 adenocarcinoma of the endometrium. Cancer Lett 248: 112-122, 2007.

18. Minagawa Y, Sato S, Ito M, Onohara Y, Nakamoto S and Kigawa J: Transvaginal ultrasonography and endometrial cytology as a diagnostic schema for endometrial cancer. Gynecol Obstet Invest 59: 149-154, 2005. 